\title{
REVERSIBLE DATABASE WATERMARKING WITH DISTORTION CONTROL
}

\author{
Seema Siledar \\ Assistant Professor, Department of Computer Science and Engineering, Marathwada Institute of Technology, \\ Aurangabad, Maharashtra, 431005, India \\ seema.siledar@mit.asia \\ Dr. Sharvari Tamane \\ Professor, University Department of Information and Communication Technology, MGM University, \\ Aurangabad, Maharashtra, 431003, India \\ sharvaree73@yahoo.com
}

\begin{abstract}
Database watermarking has emerged as an effective solution to protect ownership of relational databases. Unlike images and videos, watermark insertion in databases can cause distortion leading to loss of data quality. In this paper, we propose a reversible database watermarking technique with distortion control. Firstly, we need to select optimal attributes for applying difference expansion. Pearson Correlation Coefficient (PCC) is employed to find a pair of attributes with the highest correlation. This ensures controlled distortion in the database. Later, difference expansion is applied on the selected attributes. It makes the method reversible and original data can be recovered whenever required. Experimental results show that the proposed method is robust against insertion, deletion, and modification attacks.
\end{abstract}

Keywords: Database watermarking, reversible, distortion control, ownership protection, robust, database attacks

\section{Introduction}

Due to the increasing use of the internet and cloud computing, it is very easy to get the required database anywhere anytime. On the other hand, it has become very difficult to protect databases from unauthorized access. Moreover, once the database is shared with users over the internet, ownership protection becomes a tedious task. Also, the shared database can be changed or redistributed without the knowledge of the owners.

Database watermarking can play a vital role to prove ownership of data. This term was first coined by (Agarwal \& Kiernan, 2002). Since then, several techniques have been introduced. (Zhang, Yang, \& Niu, 2006) were the first to put forward the idea of reversible watermarking for relational databases. (Gupta \& Pieprzyk, 2009 ) proposed the use of difference expansion to watermark relational databases. Their technique was reversible as well as blind. (Farfoura, et al., 2012) suggested a reversible watermark embedding technique called predictionerror expansion on integers. (Jawad \& Khan, 2013) proposed the idea of difference expansion along with the use of genetic algorithm to reduce distortion. (Chang, Nguyen, \& Lin, 2013) put forward the idea of a reversible embedding algorithm based on histogram shifting of adjacent pixel difference. (Franco-Contreras, Coatrieux, Cuppens, Cuppens-Boulahia, \& Roux, 2014) proposed a scheme that modulates the relative angular position of the circular histogram center of mass of one numerical attribute for message embedding. (Khanduja, Verma, \& Chakraverty, 2015) suggested the idea of using a bacterial foraging algorithm for securely embedding watermark bits. (Iftikhar, Kamran, \& Anwar, 2015) proposed a robust and reversible technique for numerical databases with the use of low mutual information to select feature for watermarking.

(Imamoglu, Ulutas, \& Ulutas, 2017) suggested a reversible approach of utilizing firefly algorithm with difference expansion to embed a watermark into relational databases. (Hu, Zhao, \& Zheng, 2019) proposed a robust and reversible database watermarking technique that incorporates genetic algorithm and histogram shifting for numerical relational database. (Zhao, Jiang, \& Duan, 2019) introduced a differential evolution algorithm for selection of the optimal watermark embedding position in database. (Li, Wang, \& Luo, 2020) proposed a reversible database watermarking in which a watermark is embedded by histogram non-redundant shifting method. (Ge, et al., 2020) suggested the use of a random forest algorithm to select important attributes for watermark embedding along with genetic algorithm. (Unnikrishnan \& Pramod, 2021) proposed a system that uses a set of watermark bits to make a validation and recovery mechanism for database authentication. 


\section{Preliminaries}

We present preliminaries of the proposed work in this section.

\subsection{Pearson Correlation Coefficient (PCC)}

Pearson Correlation Coefficient (PCC) is the measure of linear correlation between two variables $x$ and $y$. It is the ratio between the covariance of two variables and the product of their standard deviations as in Eq. (1)

$$
\mathrm{r}=\frac{n\left(\sum x y\right)-\left(\sum x\right)\left(\sum y\right)}{\sqrt{\left[n \sum x^{2}-\left(\sum x\right)^{2}\right]\left[n \sum y^{2}-\left(\sum y\right)^{2}\right]}}
$$

It has a value between -1 and +1 with -1 representing the lowest correlation between the variables $x$ and $y$ whereas +1 represents the highest correlation between the variables $x$ and $y$.

\subsection{Difference Expansion}

The difference expansion method for relational databases was first proposed by (Gupta \& Pieprzyk, 2009). This method focuses on modifying attribute values by calculating their difference. Consider any two numeric attributes $A_{1}$ and $A_{2}$ present in the database. The average and difference are calculated as shown in Eq. (2) and Eq. (3)

$$
\begin{aligned}
& \text { avg }=\left\lfloor\frac{A_{1}+A_{2}}{2}\right\rfloor \\
& \operatorname{diff}=A_{1}-A_{2}
\end{aligned}
$$

Assume the watermark bit to be inserted is $b$ and calculate the new difference diff' as shown in Eq. (4)

$$
\operatorname{diff} f^{\prime}=2 * \operatorname{diff}+b
$$

Modified attribute values denoted by $A_{1}^{\prime}$ and $A_{2}^{\prime}$ are computed as in Eq. (5) and Eq. (6)

$$
\begin{aligned}
& A_{1}^{\prime}=a v g+\left\lfloor\frac{\text { diff }}{2}+1\right. \\
& A_{2}^{\prime}=\operatorname{avg}-\left\lfloor\frac{\text { diff }}{2}\right\rfloor
\end{aligned}
$$

To recover the original values of attributes $A_{1}$ and $A_{2}$, calculate the average and difference as shown in Eq. (7) and Eq. (8)

$$
\begin{aligned}
& \operatorname{avg} g^{\prime}=\left\lfloor\frac{A_{1}^{\prime}+A_{2}^{\prime}}{2}\right\rfloor \\
& \operatorname{diff} f^{\prime}=A_{1}^{\prime}-A_{2}^{\prime}
\end{aligned}
$$

The watermark bit $b$ can be extracted as in Eq. (9)

$$
b=\operatorname{diff} f^{\prime}-2\left\lfloor^{\text {diff }^{\prime}} / 2\right\rfloor
$$

Original attribute values denoted by $A_{1}$ and $A_{2}$ are computed as in Eq. (10) and Eq. (11)

$$
\begin{aligned}
& A_{1}=a v g^{\prime}+\left\lfloor\frac{\left(\text { diff }^{\prime} / 2 \mid\right)+1}{2}\right\rfloor \\
& A_{2}=a v g^{\prime}-\left\lfloor\frac{\left.\mid \text { diff }_{2} / 2\right\rfloor}{2}\right\rfloor
\end{aligned}
$$

For example, let $A_{1}=53$ and $A_{2}=58$, then, avg $=\left\lfloor\frac{53+58}{2}\right\rfloor=55.5 \approx 55$ and diff $=53-58=-5$. Assume $b=0$ for all the even position tuples and $b=1$ for the odd position tuples. Consider an even position tuple, so, the new difference is calculated as diff $f^{\prime}=2 *(-5)+0=-10$ by inserting 0 as watermark bit. Now, the modified attribute values are calculated by using Eq. (5) and Eq. (6):

$$
\begin{aligned}
& A_{1}^{\prime}=55+\left\lfloor\frac{-10+1}{2}\right\rfloor=55+\left[\frac{-9}{2}\right\rfloor=55+(-5)=50 \\
& A_{2}^{\prime}=55+\left\lfloor\frac{-10}{2}\right\rfloor=55-(-5)=55+5=60
\end{aligned}
$$

To recover the original attribute values, calculate the average and difference from the modified attribute values as avg $^{\prime}=\left[\frac{50+60}{2}\right]=55$ and diff $=50-60=-10$. Using Eq. (9), extract watermark bit $b$ as: $b=-10-$ $2\left\lfloor^{-10 / 2}\right\rfloor=-10-2(-5)=-10+10=0$. Original attribute values are recovered with the help of Eq. (10) and Eq. (11): 


$$
\begin{aligned}
& A_{1}=55+\left\lfloor\frac{(\lfloor-10 / 2\rfloor)+1}{2}\right\rfloor=55+\left\lfloor\frac{-5+1}{2}\right\rfloor=55+\frac{-4}{2}=55-2=53 \\
& A_{2}=55-\left\lfloor\frac{(\lfloor-10 / 2\rfloor)}{2}\right\rfloor=55-\left\lfloor\frac{-5}{2}\right\rfloor=55-\lfloor-2.5\rfloor=55+3=58
\end{aligned}
$$

\section{Methodology}

The proposed method is divided into two phases: Watermark Insertion and Watermark Extraction. Fig. 1 shows the various steps involved in watermark insertion phase and Fig. 2 depicts watermark extraction phase.

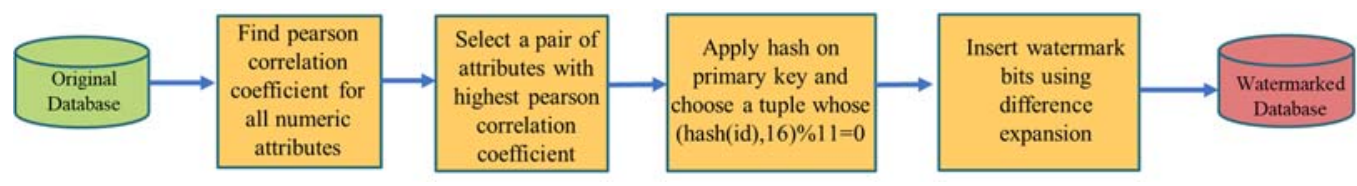

Fig. 1 Watermark Insertion

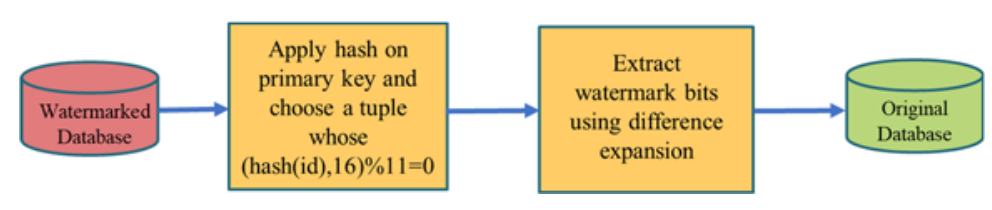

Fig. 2 Watermark Extraction

The proposed method has following features:

- Robustness: Watermark remains unaltered despite the modifications made to the database.

- Blindness: Watermark extraction does not require the knowledge of original database as well as watermark information.

- Reversible: The original database can be recovered after extracting the watermark from the watermarked database.

- Distortion control: The selection of a pair of attributes with highest PCC ensures lower distortion.

\subsection{Watermark Insertion}

During this phase, watermark bits are inserted into specific positions in the database. These bits can later be extracted to prove database ownership. The following steps are performed to insert watermark bits into the original database:

Step 1: Find Pearson Correlation Coefficient (PCC) for all the numeric attributes using Eq. (1)

Step 2: Select a pair of attributes whose Pearson Correlation Coefficient (PCC) is highest among all

Step 3: Apply hash on the primary key and choose a tuple whose (hash(id), 16) $\% 11=0$

Step 4: Insert the watermark bits in selected tuples using difference expansion

In this step, we calculate the average and difference between a pair of attributes that are selected in step 2 as follows:

$$
\begin{aligned}
& \operatorname{avg}=\left\lfloor\frac{A_{1}+A_{2}}{2}\right\rfloor \\
& \text { diff }=A_{1}-A_{2}
\end{aligned}
$$

Determine the watermark bit to be inserted using Eq. (18)

$$
b=\operatorname{diff} \& 1
$$

Compute the new difference diff' as shown in Eq. (19) by assuming the watermark bit $b$ to be inserted.

$$
\operatorname{diff} f^{\prime}=2 * \operatorname{diff}+b
$$

Modified attribute values denoted by $A_{1}^{\prime}$ and $A_{2}^{\prime}$ are computed as in Eq. (20) and Eq. (21)

$$
\begin{aligned}
& A_{1}^{\prime}=a v g+\left\lfloor\frac{\text { dif }^{\prime}+1}{2}\right\rfloor \\
& A_{2}^{\prime}=\operatorname{avg}-\left\lfloor\frac{\text { diff }}{2}\right\rfloor
\end{aligned}
$$

The input of this phase is the original database, and the output is the watermarked database. 


\subsection{Watermark Extraction}

During watermark extraction phase, the same pair of attributes that were selected in step 2 of watermark insertion phase are considered. The following steps are performed to extract watermark bits from the watermarked database:

Step 1: Apply hash on the primary key and choose a tuple whose (hash(id), 16) \% 11=0

Step 2: Extract the watermark bits from selected tuples using difference expansion

In this step, we calculate the average and difference as shown in Eq. (22) and Eq. (23)

$$
\begin{aligned}
& \operatorname{avg}^{\prime}=\left\lfloor\frac{A_{1}^{\prime}+A_{2}^{\prime}}{2}\right\rfloor \\
& \text { diff } f^{\prime}=A_{1}^{\prime}-A_{2}^{\prime}
\end{aligned}
$$

The watermark bit $b$ can be extracted using in Eq. (24)

$$
b=\operatorname{diff} f^{\prime}-2 *\left[\text { diff }^{\prime} / 2\right\rfloor
$$

Original attribute values denoted by $A_{1}$ and $A_{2}$ are recovered with the help of Eq. (25) and Eq. (26)

$$
\begin{aligned}
& A_{1}=a v g^{\prime}+\left\lfloor\frac{\left(\left\lfloor\text { diff }^{\prime} / 2\right\rfloor+1\right.}{2}\right\rfloor \\
& A_{2}=a v g^{\prime}-\left\lfloor\frac{\lfloor\text { diff } / 2\rfloor}{2}\right\rfloor
\end{aligned}
$$

The input of this phase is the watermarked database, and the original database is recovered as a result.

\section{Results and Discussion}

Experiments were conducted on the system with Intel Core i3 CPU of 2.00GHz and 4GB RAM. Indian Liver Patient Dataset with 583 instances is the test database. The dataset contains 10 variables that are Age, Gender, Total Bilirubin, Direct Bilirubin, Alkaline Phosphatase, Alamine Aminotransferase, Aspartate Aminotransferase, Total Proteins, Albumin, Albumin and Globulin Ratio. An attribute ID has been added as primary key with values ranging from 1 to 583. Gender is a categorical attribute, so it is not considered for calculating Pearson Correlation Coefficient (PCC).

For simplicity, the attributes ID, Age, Total Bilirubin, Direct Bilirubin, Alkaline Phosphatase, Alamine Aminotransferase, Aspartate Aminotransferase, Total Proteins, Albumin, Albumin and Globulin Ratio are renamed as $A_{1}, A_{2}, A_{3}, A_{4}, A_{5}, A_{6}, A_{7}, A_{8}, A_{9}$, and $A_{10}$. The Pearson Correlation Coefficient (PCC) for all the numeric values are as shown in Table 1 . The attributes $\mathrm{A}_{6}$ and $\mathrm{A}_{7}$ i.e., Alamine Aminotransferase and Aspartate Aminotransferase have the highest PCC value 0.791966 as highlighted Table 1 . Higher the PCC, lower the distortion. $10 \%$ of the total tuples i.e., 59 out of 583 , are watermarked.

\begin{tabular}{|l|c|c|c|c|c|c|c|c|c|c|}
\hline & $\mathbf{A}_{\mathbf{1}}$ & $\mathbf{A}_{\mathbf{2}}$ & $\mathbf{A}_{\mathbf{3}}$ & $\mathbf{A}_{\mathbf{4}}$ & $\mathbf{A}_{\mathbf{5}}$ & $\mathbf{A}_{\mathbf{6}}$ & $\mathbf{A}_{\mathbf{7}}$ & $\mathbf{A}_{\mathbf{8}}$ & $\mathbf{A}_{\mathbf{9}}$ & $\mathbf{A}_{\mathbf{1 0}}$ \\
\hline $\mathbf{A}_{\mathbf{1}}$ & 1 & & & & & & & & & \\
\hline $\mathbf{A}_{\mathbf{2}}$ & -0.052385 & 1 & & & & & & & & \\
\hline $\mathbf{A}_{\mathbf{3}}$ & 0.1015259 & 0.011763 & 1 & & & & & & & \\
\hline $\mathbf{A}_{\mathbf{4}}$ & 0.1362854 & 0.007529 & 0.874618 & 1 & & & & & & \\
\hline $\mathbf{A}_{\mathbf{5}}$ & -0.078805 & 0.080425 & 0.206669 & 0.234939 & 1 & & & & & \\
\hline $\mathbf{A}_{\mathbf{6}}$ & -0.12486 & -0.08688 & 0.214065 & 0.233894 & 0.125680 & 1 & & & & \\
\hline $\mathbf{A}_{\mathbf{7}}$ & -0.094106 & -0.01991 & 0.237831 & 0.257544 & 0.167196 & $\mathbf{0 . 7 9 1 9 6 6}$ & 1 & & & \\
\hline $\mathbf{A}_{\mathbf{8}}$ & 0.189634 & -0.18746 & -0.0081 & -0.000139 & -0.028514 & -0.042518 & -0.025645 & & 1 & \\
\hline $\mathbf{A}_{\mathbf{9}}$ & 0.0656466 & -0.26592 & -0.22225 & -0.228531 & -0.165453 & -0.029742 & -0.085290 & 0.784053 & 1 & \\
\hline $\mathbf{A}_{\mathbf{1 0}}$ & -0.024123 & -0.21853 & -0.20595 & -0.199745 & -0.235087 & -0.002821 & -0.070033 & 0.239690 & 0.691239 & 1 \\
\hline
\end{tabular}

Table 1. Pearson Correlation Coefficient (PCC) for all numeric attributes

\subsection{Robustness Analysis}

Robustness can be demonstrated with the help of different types of attacks on the database. We consider three types of attacks: insertion, deletion, and modification. Suppose an attacker attempts to insert, delete, or modify the tuples in the database. Experiments are conducted to simulate all these attacks with attack rate of $10 \%, 20 \%$, up to $90 \%$. Fig. 3 represents attack ratio on $\mathrm{X}$-axis and watermark detection rate on $\mathrm{Y}$-axis. 
The first experiment is performed to demonstrate insertion attack. We compute the hash function of the primary key and based on its value the tuples are selected for watermarking. Whenever any new tuple is added into the database, it will not tamper the existing watermark. As the watermark is determined by the primary key, it will remain intact even though the attacker tries to insert any number of new tuples. Thus, the proposed method is robust to insertion attack as shown in Fig. 3

The second experiment is conducted to determine the robustness of the proposed method under deletion attack. In this type of attack, the attacker attempts to randomly delete tuples from the database. As we increase the attack rate from $10 \%$ to $90 \%$ by deleting 59 tuples to 525 tuples respectively, the watermark detection rate decreases from $94.91 \%$ to $10.16 \%$. It means that the chances of watermark detection go on decreasing as we increase the deletion attack percentage. It can be observed in Fig. 3. Moreover, it is not possible to recover the complete watermark if many tuples that contain watermark information are deleted.

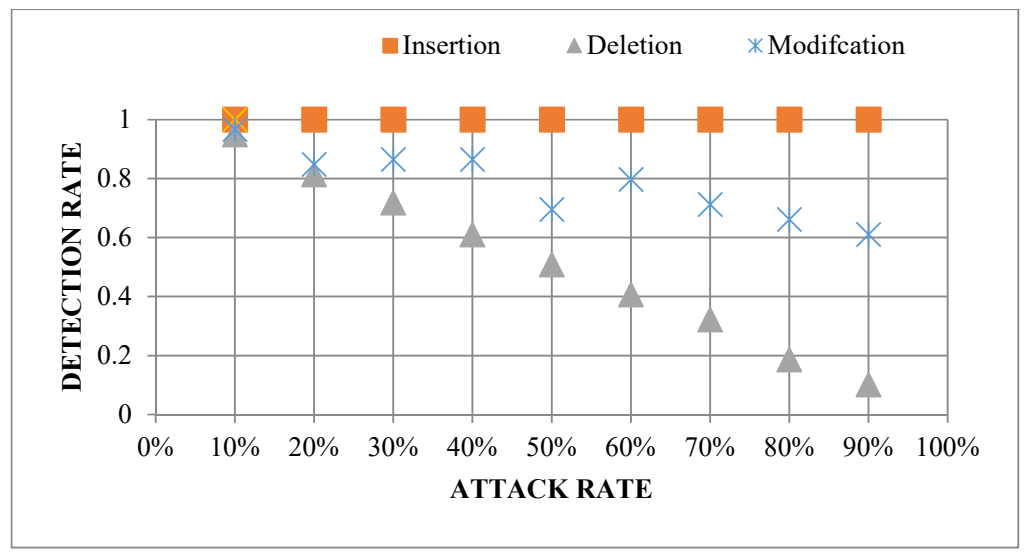

Fig. 3 Watermark Detection Rate

The third experiment is performed to demonstrate modification attack. In modification attack, the attacker attempts to randomly modify tuples in the database. Fig. 3 shows that the increase in attack rate from $10 \%$ to $90 \%$ leads to detection rate of $96.61 \%$ to $61.01 \%$ respectively. It means that there are high chances of watermark detection even with greater attack rates. From Fig. 3, it can be observed that around $60 \%$ of the watermark can be recovered even in case of $90 \%$ modification attack.

\subsection{Statistical Distortion}

The data quality of the proposed algorithm is evaluated with statistical distortion. We measure the statistical distortion through mean absolute error and variations of mean and standard deviation between the attributes before and after watermark insertion.

\subsubsection{Mean Absolute Error (MAE)}

Mean Absolute Error (MAE) can be calculated as:

$$
M A E=\frac{\sum_{i=1}^{n}\left|A_{i}-A_{i}^{w}\right|}{n}
$$

where, $\mathrm{n}$ is the total number of tuples in the database, $\mathrm{A}_{\mathrm{i}}$ is the attribute of original database and $A_{i}^{w}$ is the attribute of watermarked database.

\begin{tabular}{|c|c|}
\hline Attribute name & MAE \\
\hline Alamine Aminotransferase $\left(\mathrm{A}_{6}\right)$ & 1.63 \\
\hline Aspartate Aminotransferase $\left(\mathrm{A}_{7}\right)$ & 1.63 \\
\hline
\end{tabular}

Table 2. Mean Absolute Error (MAE)

Table 2 shows the mean absolute error for the selected attributes is 1.63 . It will be same for the pair of selected attributes because of difference expansion.

\subsubsection{Mean and Standard Deviation}

Table 3 provides the mean and standard deviation obtained for the selected attributes Alamine Aminotransferase and Aspartate Aminotransferase. These measures are computed for the original as well as the watermarked database as seen in Table 3. 


\begin{tabular}{|c|c|c|c|c|}
\hline \multirow{2}{*}{ Attribute name } & \multicolumn{2}{|c|}{ Original Database } & \multicolumn{2}{c|}{ Watermarked Database } \\
\cline { 2 - 5 } & Mean & Std & Mean & Std \\
\hline Alamine Aminotransferase $\left(\mathrm{A}_{6}\right)$ & 80.71 & 182.62 & 82.28 & 144.35 \\
\hline Aspartate Aminotransferase $\left(\mathrm{A}_{7}\right)$ & 109.91 & 288.91 & 114.43 & 247.78 \\
\hline
\end{tabular}

Table 3. Mean and Standard Deviation

To see the change of mean and standard deviation, the difference in mean and the difference in standard deviation for the watermarked attributes are calculated as in Eq. (28) and Eq. (29):

$$
\begin{aligned}
& \text { Difference in mean }=\mid \text { Mean }_{D b}-\text { Mean }_{W D b} \mid \\
& \text { Difference in standard deviation }=\left|S t d_{D b}-S t d_{W d b}\right|
\end{aligned}
$$

where, MeanDb and StdDb represent the mean and standard deviation of the original database. MeanWDb and $\mathrm{StdWDb}$ represent mean and standard deviation of the watermarked database.

\begin{tabular}{|c|c|c|}
\hline \multirow{2}{*}{ Attribute name } & \multicolumn{2}{|c|}{ Proposed method } \\
\cline { 2 - 3 } & Difference in mean & Difference in std \\
\hline Alamine Aminotransferase $\left(\mathrm{A}_{6}\right)$ & 1.57 & 38.27 \\
\hline Aspartate Aminotransferase $\left(\mathrm{A}_{7}\right)$ & 4.52 & 41.13 \\
\hline
\end{tabular}

Table 4. Difference in mean and difference in standard deviation

As shown in Table 4, the proposed method introduces minor change in mean of the selected attributes in original and watermarked database.

\section{Conclusion}

In this paper, a reversible and blind watermarking technique has been proposed for numeric relational databases. We suggested the use of Pearson Correlation Coefficient to select highly correlated attributes for applying difference expansion. Due to this, the distortion introduced in the database has a negligible effect on data quality. This is supported statistically by the difference in mean of 1.57 and 4.52 for attributes Alamine Aminotransferase (A6) and Aspartate Aminotransferase (A7) respectively. Difference in standard deviation is comparatively higher, that is, 38.47 and 41.13 for attributes A6 and A7 respectively. Experimental results show that the proposed method is robust against insertion attacks. Irrespective of the number of tuples added into the database, the watermark can still be completely recovered. Results show that the proposed method can recover around $60 \%$ of the watermark with $90 \%$ of modification attack rate. In case of deletion attack, the watermark detection rate decreases from $94.91 \%$ to $10.16 \%$ as the attack rate increases from $10 \%$ to $90 \%$. Our future work is to develop a reversible technique that will reduce distortion and increase watermark detection rate even with higher attack rates.

\section{References}

[1] Agarwal, R., \& Kiernan, J. (2002). Watermarking Relational Databases. 28th VLDB Conference, (pp. 155-166).

[2] Chang, C.-C., Nguyen, T.-S., \& Lin, C.-C. (2013). A Blind Reversible Robust Watermarking Scheme for Relational Databases. The Scientific World Journal, 1-12.

[3] Farfoura, M., Horng, S.-J., Lai, J.-L., Run, R.-S., Chen, R.-J., \& Khan, M. (2012). A blind reversible method for watermarking relational databases based on a time-stamping protocol. Expert Systems with Applications, 3185-3196.

[4] Franco-Contreras, J., Coatrieux, G., Cuppens, F., Cuppens-Boulahia, N., \& Roux, C. (2014). Robust Lossless Watermarking of Relational Databases Based on Circular Histogram Modulation. IEEE TRANSACTIONS ON INFORMATION FORENSICS AND SECURITY, 397-410.

[5] Ge, C., Sun , J., Sun, Y., Di, Y., Zhu, Y., Xie, L., \& Zhang, Y. (2020). Reversible Database Watermarking Based on Random Forest and Genetic Algorithm. 2020 International Conference on Cyber-Enabled Distributed Computing and Knowledge Discovery (CyberC). Chongqing, China: IEEE.

[6] Gupta, G., \& Pieprzyk, J. (2009). Reversible and Blind Database Watermarking Using Difference Expansion. International Journal of Digital Crime and Forensics, 12, 1-13.

[7] Hu, D., Zhao, D., \& Zheng, S. (2019). A New Robust Approach for Reversible Database Watermarking with Distortion Control. IEEE Transactions on Knowledge and Data Engineering, 1024 - 1037.

[8] Iftikhar, S., Kamran, M., \& Anwar, Z. (2015). RRW - A Robust and Reversible Watermarking Technique for Relational Data. IEEE TRANSACTIONS ON KNOWLEDGE AND DATA ENGINEERING, 1132-1145.

[9] Imamoglu, M., Ulutas, M., \& Ulutas, G. (2017). A New Reversible Database Watermarking Approach with Firefly Optimization Algorithm. Mathematical PRoblems in Engineering, 1-14.

[10] Jawad, K., \& Khan, A. (2013). Genetic algorithm and difference expansion based reversible watermarking for relational databases. Journal of Systems and Software , 2742-2753.

[11] Khanduja, V., Verma, O., \& Chakraverty, S. (2015). Watermarking relational databases using bacterial foraging algorithm. Multimedia Tools and Applications, 813-839.

[12] Li, Y., Wang, J., \& Luo, X. (2020). A reversible database watermarking method non-redundancy shifting-based histogram gaps. International Journal of Distributed Sensor Networks, 1-11. 
[13] Unnikrishnan, K., \& Pramod, K. (2021). Prediction-based robust blind reversible watermarking for relational databases. International Journal of Information and Computer Security.

[14] Zhang, Y., Yang , B., \& Niu, X.-M. (2006). Reversible Watermarking for Relational Database Authentication . Journal of Computers, 59-66.

[15] Zhao, M., Jiang, C., \& Duan, J. (2019). Reversible Database Watermarking Based on Differential Evolution Algorithm. 2019 International Conference on Artificial Intelligence and Advanced Manufacturing (AIAM). Dublin, Ireland: IEEE.

\section{Authors Profile}

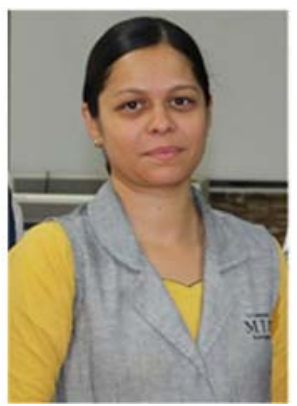

Seema Siledar received M.E. degree in Computer Science and Engineering from Government College of Engineering, Aurangabad. Currently, she is pursuing her Ph.D. from Dr. Babasaheb Marathwada University. She is an Assistant Professor in Computer Science and Engineering department at Marathwada Institute of Technology. Her research interests include privacy and security in database.

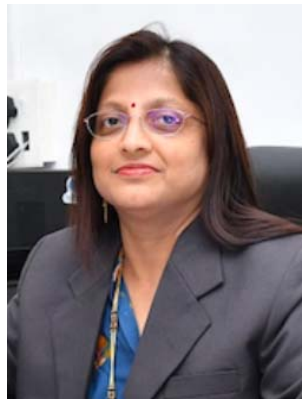

Dr. Sharvari Tamane received Ph.D. degree in Computer Science and Engineering from Dr. Babasaheb Marathwada University. Currently, she is working as Professor and Head in University Department of Information and Communication Technology at M.G.M. University. Her research interests include Big Data, Cloud Computing, Network Security. 